\title{
Concurrent Respiratory Motion Correction of Abdominal PET and Dynamic Contrast-Enhanced-MRI Using a Compressed Sensing Approach
}

\author{
Niccolo Fuin ${ }^{1}$, Onofrio A. Catalano ${ }^{1}$, Michele Scipioni ${ }^{1,2}$, Lisanne P.W. Canjels ${ }^{3}$, David Izquierdo-Garcia ${ }^{1}$, \\ Stefano Pedemonte ${ }^{1}$, and Ciprian Catana ${ }^{1}$ \\ ${ }^{I}$ Athinoula A. Martinos Center for Biomedical Imaging, Department of Radiology, Massachusetts General Hospital and Harvard \\ Medical School, Charlestown, Massachusetts; ${ }^{2}$ Department of Information Engineering, University of Pisa, Pisa, Italy; and \\ ${ }^{3}$ Department of Biomedical Engineering, Eindhoven University of Technology, Eindhoven, The Netherlands
}

We present an approach for concurrent reconstruction of respiratory motion-compensated abdominal dynamic contrast-enhanced (DCE)-MRI and PET data in an integrated PET/MR scanner. The MR and PET reconstructions share the same motion vector fields derived from radial MR data; the approach is robust to changes in respiratory pattern and does not increase the total acquisition time. Methods: PET and DCE-MRI data of 12 oncologic patients were simultaneously acquired for $6 \mathrm{~min}$ on an integrated PET/MR system after administration of ${ }^{18} \mathrm{~F}-\mathrm{FDG}$ and gadoterate meglumine. Goldenangle radial MR data were continuously acquired simultaneously with PET data and sorted into multiple motion phases on the basis of a respiratory signal derived directly from the radial MR data. The resulting multidimensional dataset was reconstructed using a compressed sensing approach that exploits sparsity among respiratory phases. Motion vector fields obtained using the full 6-min $\left(\mathrm{MC}_{6-\mathrm{min}}\right)$ and only the last $1 \mathrm{~min}\left(\mathrm{MC}_{1-\mathrm{min}}\right)$ of data were incorporated into the $\mathrm{PET}$ reconstruction to obtain motion-corrected PET images and in an MR iterative reconstruction algorithm to produce a series of motion-corrected DCE-MR images (moco_GRASP). The motioncorrection methods $\left(\mathrm{MC}_{6-\mathrm{min}}\right.$ and $\left.\mathrm{MC}_{1-\mathrm{min}}\right)$ were evaluated by qualitative analysis of the MR images and quantitative analysis of SUV $V_{\max }$ and $\mathrm{SUV}_{\text {mean }}$, contrast, signal-to-noise ratio (SNR), and lesion volume in the PET images. Results: Motion-corrected $\mathrm{MC}_{6-\min } \mathrm{PET}$ images demonstrated $30 \%, 23 \%, 34 \%$, and $18 \%$ increases in average $S_{\text {SUax }}$, SUV mean, contrast, and SNR and an average $40 \%$ reduction in lesion volume with respect to the non-motion-corrected $\mathrm{PET}$ images. The changes in these figures of merit were smaller but still substantial for the $\mathrm{MC}_{1-\min }$ protocol: $19 \%, 10 \%$, $15 \%$, and $9 \%$ increases in average SUV $_{\max }$, SUV $_{\text {mean }}$, contrast, and SNR; and a $28 \%$ reduction in lesion volume. Moco_GRASP images were deemed of acceptable or better diagnostic image quality with respect to conventional breath-hold Cartesian volumetric interpolated breath-hold examination acquisitions. Conclusion: We presented a method that allows the simultaneous acquisition of respiratory motion-corrected diagnostic quality DCE-MRI and quantitatively accurate PET data in an integrated PET/MR scanner with negligible prolongation in acquisition time compared with routine PET/DCE-MRI protocols.

\footnotetext{
Received Oct. 18, 2017; revision accepted Jan. 15, 2018.

For correspondence or reprints contact: Ciprian Catana, Athinoula A. Martinos Center for Biomedical Imaging, Massachusetts General Hospital and Harvard Medical School, 149 13th St., Charlestown, MA 02129.

E-mail: ccatana@nmr.mgh.harvard.edu

Published online Jan. 25, 2018.

COPYRIGHT (C 2018 by the Society of Nuclear Medicine and Molecular Imaging.
}

Key Words: motion-correction; PET/MRI; DCE-MRI; compressed sensing

J Nucl Med 2018; 59:1474-1479

DOI: 10.2967/jnumed.117.203943

$\mathbf{H}$ ybrid PET and MRI (PET/MR) scanners are innovative devices that allow the simultaneous acquisition of metabolic, anatomic, and physiologic data for research and clinical applications (1). For example, the uptake of ${ }^{18} \mathrm{~F}-\mathrm{FDG}$ (using PET) and changes in signal intensity after MR contrast administration (using dynamic contrast-enhanced-MRI [DCE-MRI]) can be concurrently assessed in suggestive lesions. DCE-MRI is an integral part of abdominopelvic examinations, being useful for tumor detection and characterization. For this purpose, images have to be acquired at multiple time points after the injection of the MR contrast agent. In current clinical protocols, T1-weighted MR data are acquired at each time point during 14- to 20-s breath holds over a period of 2-6 $\min (2)$, which requires patient cooperation.

In addition to improving the patient experience by performing a 1-stop-shop examination, integrated PET/MR scanners also have the potential to greatly improve the image quality by eliminating the issues related to patient repositioning and registration of separately acquired images. However, respiratory motion is still a major source of deterioration of image quality in PET and could be a cause of misregistration between PET and DCE-MR images, as PET data are acquired during free-breathing (3). To control the effects of motion, the acquired PET data can be gated based on a respiratory signal obtained with an external device (e.g., pressure sensor mounted in a chest belt) $(4,5)$. This technique, however, sacrifices signal-to-noise (SNR), as it uses only a small fraction of the emission events. Alternatively, motion compensation can be performed assuming that a respiratory signal and motion vector fields (MVFs) are available (6). Data-driven motion-compensation techniques to obtain these from the PET data itself have been developed $(7,8)$. Although useful in certain scenarios, these approaches are dependent on the counting statistics and spatial distribution of the radiotracer in the body.

As an alternative, the simultaneous acquisition of MR data using hybrid PET/MR scanners offers the possibility to estimate the MVFs by nonrigidly coregistering a series of high-temporospatial-resolution 
MR images (9). Such a method is more robust than PET-based methods and has the advantage of being radiotracer-independent. Numerous techniques have been proposed for characterizing motion using MR in the context of PET/MR (10-15). For example, the respiration model can be constructed from a series of 2-dimensional images repeatedly acquired over several respiratory cycles $(10,11)$. Tagged MR, phase-contrast MR, and pulse field gradient methods can also be used to estimate the motion fields and have been used for MR-assisted PET motion correction (1215). Although these approaches have been shown to lead to an improvement in PET image quality, they typically require several minutes of dedicated MR acquisition per bed position or use a small number of motion phases or reduced spatial resolution or restrict the registration to only 2 dimensions. Furthermore, these MR sequences are not useful for diagnostic purposes, thus requiring a change to the standard protocols to be able to collect the respiratory signal or MR-derived motion model in a clinical setting.

To foster clinical acceptance, the MR-based respiratory motioncorrection techniques must not increase the total acquisition time, be robust to changes in respiratory patterns during the whole scanning period, and provide MR images of diagnostic value. In an effort to meet these goals, the data acquired using a self-gated golden-angle 3-dimensional radial stack-of-stars MR spoiled gradient echo sequence (radial_VIBE [volumetric interpolated breath-hold examination]) $(16,17)$ was proposed for motion correction of PET images (18). Radial schemes allow for respiratory self-gating to obtain the MVFs. In addition, the resulting static T1weighted MR images provide diagnostic information $(19,20)$. However, despite these advantages, the 6- to 10-min acquisition time of the data needed for motion modeling (18) prevented the acquisition of other diagnostic MR data. When the acquisition time was reduced to under $2 \mathrm{~min}$, streak artifacts appeared from azimuthal undersampling of the radial k-space trajectory. These artifacts decrease the quality of motion estimation and thus of the resulting motion-compensated PET and MR images if no artifactresistant methods are applied $(21,22)$.

Compressed sensing (CS) reconstruction of the MR images $(21,22)$ can help to highly increase the tolerance to undersampling streaking artifact. The acquisition of a radial_VIBE MR sequence together with a CS reconstruction framework allowed the reconstruction of clinically useful DCE-MR images of the abdomen $(21,23)$. A motion signal was extracted from a radial_VIBE MR sequence (24), and distinct motion phase images were obtained from a radial_VIBE DCE-MRI sequence (25).

In this work, building on previously published methods (2125 ), we aimed to demonstrate that it is possible to perform concurrent reconstruction of respiratory motion-compensated DCE-MRI and PET data using MVFs derived from radial MR data with a CS approach. The same radial MR data are therefore used to obtain the respiratory signal, MVFs, and motion-compensated DCE-MR images. Concurrent in this context was used to denote the fact that the same MVFs were used for both reconstructions and not that the data were jointly reconstructed.

Our second goal was to demonstrate that a respiratory motion model can also be obtained from a 1-min radial_VIBE MR acquisition using a CS reconstruction. This allows the acquisition of other diagnostic MR data during PET/MR scans when a DCE-MRI is not required. As described above, this shorter MR sequence also allows us to obtain the respiratory motion signal and displacement fields to perform PET respiratory motion correction.
We investigated the feasibility and robustness of the 2 methods by performing a comparative study of the motion-corrected and uncorrected PET and of the obtained DCE-MR images, assessing the differences in radiotracer uptake quantification and lesion volume.

\section{MATERIALS AND METHODS}

\section{Data Acquisition}

PET and MR data were acquired simultaneously using the Biograph mMR scanner (Siemens Healthineers) as part of a study approved by the local Institutional Review Board. Twelve patients with suspected malignant disease in the abdomen were enrolled in this study. Emission data were acquired $108 \pm 14$ min (mean \pm SD) after administration of $351 \pm 20 \mathrm{MBq}$ of ${ }^{18} \mathrm{~F}$-FDG. The data were acquired in 3-dimensional mode for $6 \mathrm{~min}$ after running a 2-point Dixon sequence to derive the attenuation map ( $\mu$-map) during end-expiration breath hold. Concurrently, the radial_VIBE using a golden-angle acquisition scheme (16) was run, and next a conventional Cartesian volumetric interpolated breath-hold examination (bh_VIBE) sequence was also acquired. Relevant imaging parameters were axial phase encoding, repetition time/echo time $(\mathrm{TR} / \mathrm{TE}) \approx 3.75 / 1.7 \mathrm{~ms}$, flip angle $=10$, field of view $=400 \times 400 \times 258 \mathrm{~mm}^{3}$, number of readout points in each spoke $=256$, number of partitions $=56$, with $15 \%$ slice resolution reduction and 6/8 partial Fourier applied along the slice dimension, resulting in a spatial resolution of $1.56 \times 1.56 \times 4.6 \mathrm{~mm}^{3}$. The radial_VIBE sequence was acquired for 6 min during the entire PET acquisition. An intravenous injection of $10 \mathrm{~mL}$ of gadoterate meglumine (Dotarem; Guerbet LLC) was initialized $40 \mathrm{~s}$ after the start of the radial_ VIBE data acquisition, followed by a $20-\mathrm{mL}$ saline flush, both injected at a rate of $2 \mathrm{~mL} / \mathrm{s}$. The conventional bh_VIBE data were acquired $20 \mathrm{~s}$ after the end of the radial_VIBE acquisition, in the same bed position.

\section{Respiratory Motion Signal Extraction and Data Binning}

A respiratory motion signal was obtained from the radial_VIBE MR data. The stack-of-stars k-space trajectory allowed the derivation of a self-gating respiratory motion signal from the central k-space line $(\mathrm{kx}=\mathrm{ky}=0)$. Specifically, a projection profile was computed for each acquisition angle by taking a 1-dimensional partition-direction Fourier transform of the central k-space line. The projection profiles from all the radiofrequency coil elements were first concatenated. Next, principal component analysis was performed and the component with the highest peak in the frequency range of $0.1-0.5 \mathrm{~Hz}$ was selected (24). The DCE signal was estimated using a spline data-fitting procedure and subtracted from the original signal (25). Spurious highfrequency components were removed using a low-pass filter.

On the basis of the respiratory signals, the MR k-space and the PET list-mode data were subdivided into 6 respiratory bins using a variable amplitude-based method. As opposed to traditional amplitude-based gating schemes, each bin generated using the variable amplitudebased binning approach contained an equal amount of data. This scheme ensured comparable statistics for both the MR raw and the PET list-mode data.

Because the variable amplitude-based binning could be performed only if PET and MR data acquisitions were fully synchronized, the MR sequence was modified to transmit a synchronization trigger into the PET list-mode stream at every pass through the center of the k-space.

\section{Statistic MR Reconstruction of Motion Phases}

The volumes corresponding to the 6 respiratory phases were reconstructed from the radial_VIBE MR data. The reconstruction method we adopted was a combination of CS and parallel imaging, in a framework known as golden-angle radial sparse parallel MR (GRASP) (21). In this specific implementation, we used a fully 3dimensional graphics processing unit (GPU)-based non-uniform fast 
Fourier transform (NUFFT) operator $(26,27)$. As demonstrated (21), GRASP enables robust reconstruction even in the presence of strong undersampling, thus suppressing artifacts such as streak or noise when the total number of radial planes decreases. To test the robustness of the method to shorter MR acquisition times we used the full 6-min radial_VIBE acquisition $\left(\mathrm{MC}_{6-\mathrm{min}}\right)$ or only the $1 \mathrm{~min}$ of data $\left(\mathrm{MC}_{1-\mathrm{min}}\right)$ to generate the motion phases. The last minute of the acquisition was used, as in this time period, gadoterate meglumine was present in the tissues, providing better MR contrast with respect to, for example, the first minute of acquisition.

On the basis of the respiratory motion signal, a set of $6 \times 400\left(\mathrm{MC}_{6-\mathrm{min}}\right)$ and $6 \times 66\left(\mathrm{MC}_{1-\mathrm{min}}\right)$ radial $\mathrm{k}$-space planes were generated and reconstructed using the GRASP framework. For comparison, both datasets were also noniteratively reconstructed using only an inverse NUFFT operator (in the remainder of this article, these reconstructions are named $\mathrm{MC}_{6 \text {-ifft }}$ and $\mathrm{MC}_{1 \text {-ifft }}$, respectively). The end-expiration phase was set as the reference phase; the other phase volumes were registered to the reference volume using a B-spline nonrigid image registration software (Elastix; UMC Utrecht (28)), and corresponding MVFs were also obtained.

\section{PET and MR Motion-Corrected Reconstruction}

The MVFs were used by iterative reconstruction algorithms to obtain motion-corrected DCE-MRI and PET images. These algorithms reconstructed iteratively the images at a given reference respiratory phase, accounting for the deformations at every iteration.

Reconstructions of 4-dimensional (4D) respiratory motion-resolved PET images were performed using a 4D ordinary Poisson orderedsubset expectation maximization (4D OP-OSEM) algorithm (29). The updated equation of the vector of emission rates $\lambda$, at every iteration $n$, is defined as follows:

$$
\lambda_{i}^{(n+1)}=\sum_{x}\left(\frac{\lambda_{i, t^{x}}^{n}}{\sum_{d} T_{x}^{\prime} h_{i d} c_{d} a_{d t^{x}}} \sum_{d} T_{x}^{\prime} h_{i d} \frac{v_{d t^{x}}}{\sum_{j} T_{x} h_{j d} \lambda_{j}^{n}+\frac{s_{d t^{x}}}{a_{d t^{x}}}+\frac{r_{d d^{x}}}{c_{d} a_{d t^{x}}}}\right), \quad \text { Eq. } 1
$$

where the elements of the system matrix $h_{i d}$ represent the geometric probability that photons emitted from voxel $i$ are detected in the line of response (LOR) $d ; v$ are the emission data; $a_{d}$ are the attenuation factors; $c_{d}$ are the detector normalization factors; $s_{d}$ and $r_{d}$ are the expected contributions of scatter and random coincidences, respectively. Scatter distribution sinograms were calculated using an implementation of the single scatter simulation method with relative scaling, provided by the manufacturer. The standard OP-OSEM algorithm was extended to incorporate MVFs into the reconstruction process $(30,31)$. The emission data, randoms, and attenuation-correction factors are motion phase-dependent. $T_{x}$ is defined as a transforming operation, mapping the volume of motion phase $t^{1}$ to $t^{x}$, applying the dedicated MVFs (28); and $T_{x}^{\prime}$ is the inverse transforming operation, mapping the volume of motion phase $t^{x}$ to $t^{1}$. This algorithm reconstructed iteratively the image at a given reference respiratory phase, accounting for the deformations at every OSEM iteration. Each update of the image was obtained by warping the current image estimate according to the deformations at all respiratory phases, projecting the warped images (using a warped $\mu$-map), backprojecting the residuals of the projections and warping the resulting volumes back to the reference frame; the warped volumes were summed to form the new estimate of the activity. The 4D OP-OSEM algorithm naturally accounted for the deformation of the $\mu$-map and the corrections for scatter and random coincidences. The 3-dimensional static images, used for comparison, were obtained without including the transformation in the reconstruction. All the resulting images were postsmoothed using a gaussian filter with a 4-mm kernel. The algorithm was implemented using the GPU-based tomographic reconstruction software Occiput.io (32).

For the motion-corrected DCE-MRI reconstruction framework (moco_GRASP), the acquired golden-angle dataset was sorted into multiple sets of undersampled data. The radial $\mathrm{k}$-space data were sorted into 6 respiratory phases and 18 time bins. Similarly to the 4D OPOSEM algorithm, the moco_GRASP algorithm accounted for the MVFs at every iteration. The MVFs were therefore directly included into the reconstruction framework that outputs a single MR image, at the reference phase, for every time bin. A total variation constraint was enforced along the contrast-enhancement (or time) dimension (21). The MR reconstruction cost function was formulated as follows:

$$
C(f)=\operatorname{argmin} F \sum_{t} T_{x} B f-m+a|Z f|,
$$

where $F$ is the NUFFT operator (27) defined for the radial sampling pattern; $B$ represents the coil sensitivity map; $f$ is the $4 \mathrm{D}$ dynamic image series with an additional contrast-enhancement dimension; $m$ is the corresponding multicoil radial $\mathrm{k}$-space data sorted according to the 4 data dimensions; $T$ is the transforming operation defined for Equation 1 ; and $Z$ is the sparsifying transform applied to the contrast-enhancement dimension with regularization parameter $\alpha$. From the first term of Equation 2, the following updated MR image volume $f_{x}^{(i+1)}$ for motion phase $\mathrm{x}$ and iteration $(i+1)$ can be derived:

$$
f_{x}^{(i+1)}=f_{x}^{i}+\beta\left(F^{\prime} T_{x}^{\prime}\left(F T_{x} B f^{i}-m_{x}\right)\right)
$$

The obtained volumes for the 6 phases were summed, at every iteration, to form the new estimates of the DCE-MR images $f^{(i+1)}$. This algorithm reconstructed iteratively the dynamic MR images at a given reference respiratory phase, using $100 \%$ of the MR raw data.

\section{Analysis of Impact of Motion Correction on PET and MR Image Quality and Quantification}

Analysis of the MR Respiratory Phase Images Used to Generate MVFs. The MR volumes corresponding to the 6 respiratory phases were reconstructed using both the GRASP method and the inverse

\section{TABLE 1}

MSE for Different Reduced Scan Times and for Different Reconstruction Methods, with Respect to Full 6-Minute Acquisition Using GRASP Reconstruction

\begin{tabular}{lccc}
\hline Patient & MC $_{6 \text {-ifft }}$ & MC $_{\text {1-ifft }}$ & 1-min GRASP \\
\hline 1 & 219 & 2073 & 956 \\
2 & 178 & 1413 & 463 \\
3 & 184 & 1666 & 287 \\
4 & 155 & 1162 & 218 \\
5 & 221 & 1845 & 489 \\
6 & 165 & 1940 & 938 \\
7 & 205 & 1172 & 309 \\
8 & 147 & 1210 & 256 \\
9 & 179 & 1751 & 429 \\
10 & 134 & 1202 & 293 \\
11 & 222 & 1804 & 460 \\
12 & 178 & 1399 & 483 \\
\hline
\end{tabular}




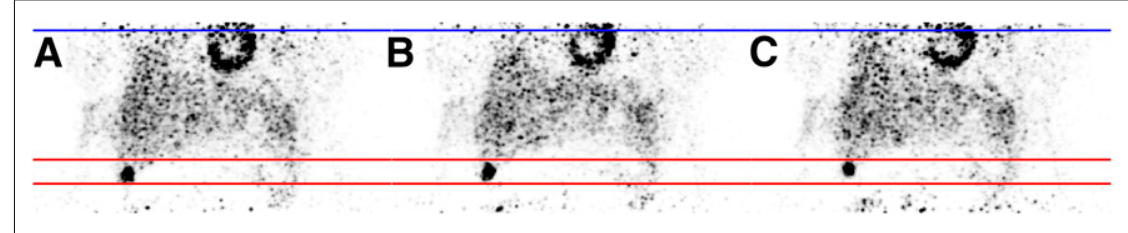

FIGURE 1. PET reconstructions for different respiratory gates, showing a coronal slice through a lesion in liver of patient 1. (A) End-of-inspiration motion phase. (B) Intermediate phase. (C) End-ofexpiration phase.

NUFFT operator from the 6- and 1-min acquisitions. The images were first qualitatively analyzed with a focus on the presence of streak artifacts. Next, the mean square errors (MSEs) were calculated, to compare the images obtained using the reduced acquisition time and for the different reconstruction methods with the images obtained from the full 6-min acquisition and using the GRASP reconstruction as reference.

PET Image Analysis. For each patient, MVFs estimated from the MR volumes corresponding to the 6 respiratory phases, resampled to the PET resolution and field of view, were used to generate motioncorrected PET images as explained in the previous section. It is important to note that the various MVFs were applied to the same gated PET sinograms. The motion-corrected PET images were quantitatively compared with the uncorrected ones by assessing changes in SUVs in lesions that exhibited tracer uptake sufficient for unambiguous segmentation in all static, gated, and motioncorrected images. Eighteen lesions were identified, and isocontour volumes of interest were segmented for each lesion individually using a region-growing algorithm (isocontour threshold, 50\%). The $\mathrm{SUV}_{\max }$ and $\mathrm{SUV}_{\text {mean }}$ in each isocontour volume of interest were calculated (33). Contrast (C), SNR, and lesion volume were also calculated as previously described (8). Image noise was obtained as the SD in a cubical volume of interest defined in the liver excluding voxels at the edge or those with focal tracer uptake.

DCE-MR Image Analysis. DCE-MRI data were obtained simultaneously with the PET data acquisition using the moco_GRASP reconstruction approach. A set of 18 pre- and postcontrast enhancement images were generated from the DCE-MRI data for every patient. The images corresponding to the baseline, early arterial, dominant arterial, portal venous, equilibrium, and late phases were selected. A comparative analysis of the image quality obtained with the conventional bh_VIBE acquisition and moco_GRASP reconstruction was its full amplitude range.

possible only for the late phase of contrast enhancement images, as bh_VIBE images were acquired 3,560 $\mathrm{s}$ after contrast injection (20 s after the end of the radial_VIBE acquisition). The resulting images were visually evaluated by an accredited radiologist with $18 \mathrm{y}$ of experience in clinical oncology.

\section{RESULTS}

\section{Morphologic MR Image Analysis}

The MR images for the end-expiratory and end-inspiratory phases for patient 1 are shown in Supplemental Figure 1 (supplemental materials are available at http://jnm. snmjournals.org). The MR images obtained from the 6-min acquisition using GRASP reconstruction $\left(\mathrm{MC}_{6-\mathrm{min}}\right)$ are presented in Supplemental Figures 1A and 1B. The image quality for the 1min acquisition using GRASP reconstruction $\left(\mathrm{MC}_{1-\mathrm{min}}\right)$ appeared stable, and only local artifacts were noticed visually in Supplemental Figures 1C and 1D. A substantial decrease in image quality can be observed in the images reconstructed using the noniterative method (Supplemental Figs. 1E and 1F). MSEs for the different reduced scan times and for the different reconstruction methods, with respect to the full 6-min acquisition using GRASP reconstruction, are given in Table 1 . With a reduced scan time of $1 \mathrm{~min}$ and $\mathrm{MC}_{1-\mathrm{ifft}}$ reconstruction, strong deviations are introduced. With a scan time of $1 \mathrm{~min}$ and GRASP reconstruction, deviations are reduced by $70 \%$ but they still do not reach the same level of a 6-min acquisition with a simple inverse FFT reconstruction. Differences in the deformation fields, used for PET motion correction and obtained with $\mathrm{MC}_{1-\min }$ (with respect to $\mathrm{MC}_{6-\mathrm{min}}$ ), do not only originate from the increased artifact level, but may also be attributed to a change in the respiratory pattern, for example, if the patient takes deeper breaths after injection of gadolinium. With a 1-min acquisition, the breathing pattern of the patient is not followed continuously and may not be captured in

\section{Analysis of Motion-Corrected PET Data}

The PET images corresponding to 3 respiratory gates for a representative patient are shown in Figure 1. The respiration-induced motion of the liver lesion in the craniocaudal direction can be appreciated. Examples of the uncorrected and motion-corrected PET images using both motion-correction protocols $\left(\mathrm{MC}_{1-\min }\right.$ and $\left.\mathrm{MC}_{6-\min }\right)$ are given

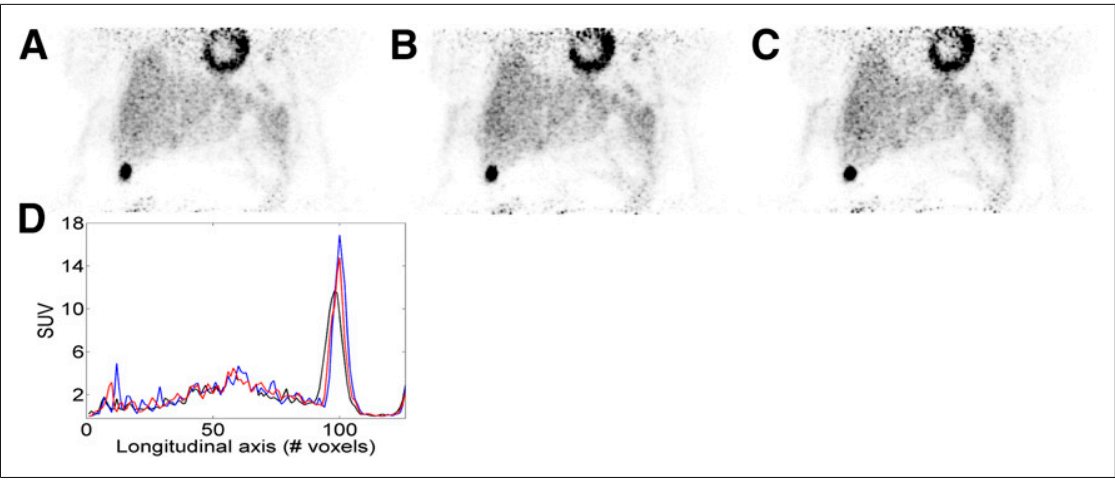

FIGURE 2. Comparison of uncorrected $(A), M C_{1-\min }(B)$, and $M C_{6-m i n}(C) P E T$ reconstructions for patient 1; showing coronal slice presented in Figure 1. The plots in $D$ show line profiles through lesion for the 3 reconstructions methods: black $=$ non-motion-corrected; red $=\mathrm{MC}_{1 \text {-min }}$; blue $=\mathrm{MC}_{6-\mathrm{min}}$. in Figure 2. The improved sharpness when compared with the uncorrected images can be appreciated visually and from the line profile defined across the lesion (Fig. 2D). For this specific lesion, the $\mathrm{SUV}_{\text {peak }}$ value was increased by $45 \%$ using $\mathrm{MC}_{6-\min }$ and $29 \%$ using $\mathrm{MC}_{1-\min }$. Similar images for a different patient are shown in Supplemental Figures 2 and 3. The results of the quantitative analysis for all the patients are presented in the bar charts of Figure 3. The motion-corrected $\mathrm{MC}_{6-\text { min }}$ PET images exhibited the highest $\mathrm{SUV}_{\text {mean }}, \mathrm{SUV}_{\max }$, contrast, and SNR, with average relative differences of $\mathrm{dSUV}_{\text {max }}=30 \%$, $\mathrm{dSUV}_{\text {mean }}$ $=23 \%$, dContrast $=34 \%, \mathrm{dSNR}=18 \%$, 


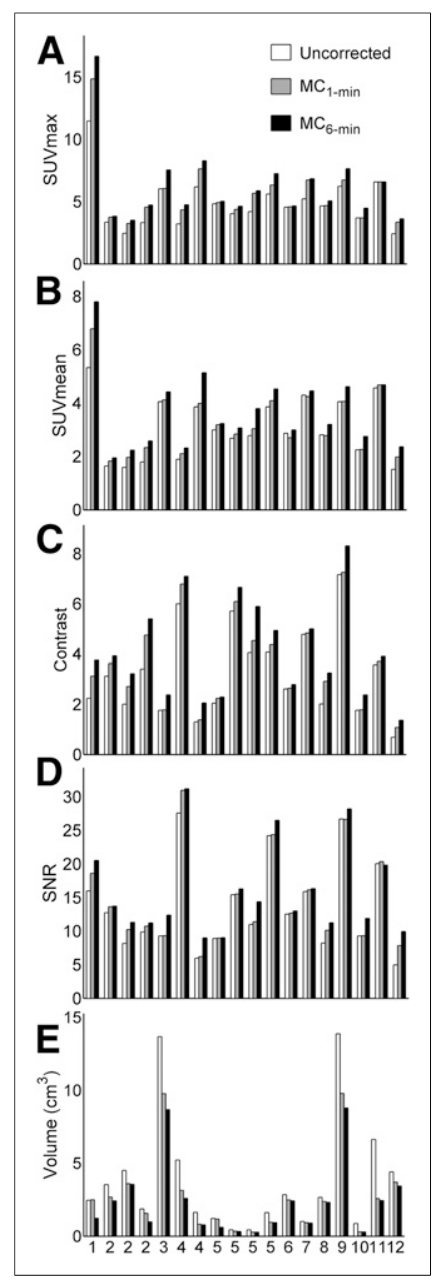

FIGURE 3. Bar charts representing figures of merit for quantification of motion-compensation accuracy for $\mathrm{MC}_{1-\min }$ and $\mathrm{MC}_{6-\min }$ protocols with respect to non-motion-corrected PET images. (A) Differences in SUV $V_{\text {max }}$ (B) Differences in SUV $V_{\text {mean }}$. (C) Differences in contrast. (D) Differences in SNR. (E) Differences in lesion volume (in $\mathrm{cm}^{3}$ ).

mental Figs. 5A and 5C) and with bh VIBE (Supplemental Figs. 5B and 5D). From these examples, it can be noted that the MR images obtained with moco_GRASP are slightly less sharp than the images obtained from a bh_VIBE acquisition. However, the free-breathing moco_GRASP acquisition is robust to motion and does not present motion artifact that could occur when the patient is incapable of breath-holding during a 20-s bh_VIBE acquisition (Supplemental Fig. 5D).

\section{DISCUSSION}

Respiratory motion is one of the primary challenges in achieving unbiased measurements of lesion radiotracer concentration in abdominal PET imaging and a source of misregistration between the PET and DCE-MR images even in simultaneous PET/MRI.

In this study, we proposed a method for concurrent reconstruction of respiratory motion-compensated liver DCE-MRI and PET data. The motion model is derived from continuously acquired radial
DCE-MRI data (16) using the proposed protocol $\left(\mathrm{MC}_{6-\mathrm{min}}\right)$, and the different motion phases are reconstructed using a $\mathrm{CS}$ approach (25). The acquisition time of the DCE-MRI data that also allows the derivation of the respiratory motion model is similar to that of the current clinical protocol that requires a set of Cartesian bh_VIBE DCE-MRI acquisitions, but our approach follows continuously the breathing pattern of the patient during acquisition. To allow the derivation of the motion model while minimally modifying the clinical MR protocol even in those studies that do not require DCE-MRI, we also proposed a 1-min data acquisition protocol for respiratory motion characterization that could be appended to routine examinations.

Improvements of PET image quality and quantification accuracy in terms of $\mathrm{SUV}_{\text {max }}, \mathrm{SUV}_{\text {mean }}$, contrast, and SNR were demonstrated in all 12 patients. These values were similar to those reported in previous studies (e.g., 12\%-19\% SUV underestimation) $(8,18)$ for the $\mathrm{MC}_{1 \text {-min }}$ protocol and even higher (up to $30 \%$ ) for the $\mathrm{MC}_{6-\text { min }}$ protocol. Impact on patient management, for a greater pool of patients, will be demonstrated in a future study. The better performance of the $\mathrm{MC}_{6-\mathrm{min}}$ protocol with respect to previously presented results $(8,10,11,18)$ can be attributed to several factors related to the MR acquisition and to the adopted MR reconstruction method. The $\mathrm{MR}$ acquisition in $\mathrm{MC}_{6-\min }$ was 6 min long, yielding better SNR, improved sharpness, and reduced streak artifacts in the MR images corresponding to the respiratory phases. The presented method follows continuously the respiratory pattern for the entire PET acquisition and produces a comprehensive motion model. Furthermore, for the first time, DCE-MR images have been used to obtain a motion model, allowing for a much better delineation of the lesion from the adjacent healthy tissue. The contrast enhancement, consequently, produced an improved motion model for the lesion itself. Finally, the use of iterative GRASP reconstruction (21) to obtain MR images of the motion phases helps to increase the tolerance to undersampling artifacts.

Building on previously published methods (21-25), we also introduced a reconstruction method to obtain motion-corrected DCE-MR images of the abdomen, from radial VIBE data. In the study of Feng et al. (25), continuously acquired k-space data were sorted into multiple sets of undersampled datasets with distinct motion states, using motion signal extracted directly from DCEMRI data (24). The motion phases were then reconstructed relying on the use of CS. In the work presented in this article, MVFs are

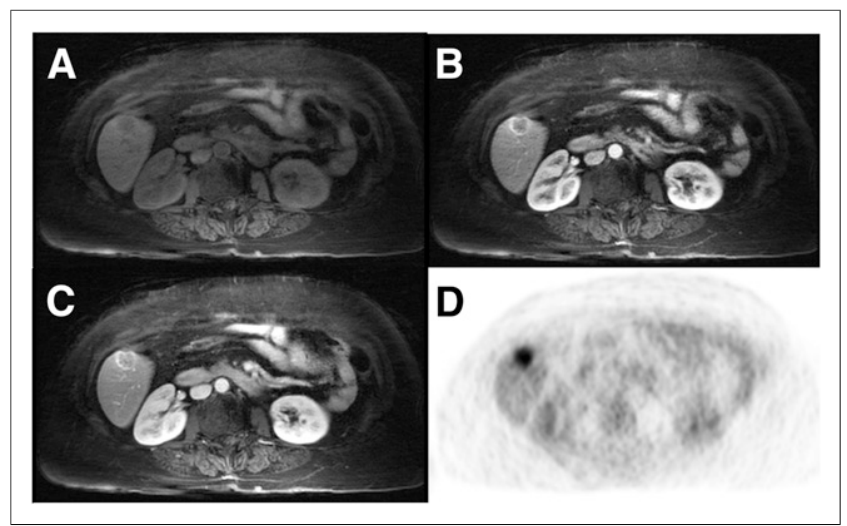

FIGURE 4. DCE-MR images of liver using moco_GRASP reconstruction $(A-C)$, together with corresponding motion-corrected PET image (D), showing axial slice through lesion in liver of patient 1. (A) Noncontrast-enhanced phase. (B) Arterial dominant phase. (C) Portal venous phase. (D) Corresponding motion-corrected PET image. 
extracted from the reconstructed motion phases and subsequently included in a 4D MRI statistical reconstruction process to obtain motion-corrected DCE-MR images. The multiphase liver images were deemed of diagnostic quality by an accredited radiologist. Slightly lower image quality for the arterial compared with the venous phase was observed as previously reported (23). This is likely related to increased streak artifacts from the rapid change in signal intensity in the aorta and liver from the arrival of the bolus of contrast, but, in all cases, images were considered as acceptable or qualitatively better for all phases of enhancement.

The $\mathrm{MC}_{1-\min }$ approach might be limited in cases for which the breathing pattern and the maximum breathing amplitude of a patient change considerably during the remaining PET acquisition. Moreover, when shortening the MR scan, the physiologic signal would need to be derived by mean of an external device (e.g., respiratory belt) or using PET-based self-gating $(7,8)$.

Finally, dedicated hardware and software are required for the PET and MR data processing and image reconstructions. To reduce the computational effort, the DCE-MRI reconstruction software was implemented using a fully 3-dimensional GPU-based NUFFT operator (27). The PET motion-corrected reconstructions were also implemented using GPU-based projector and back-projector operators (32). A package containing the necessary software will be included in the GPU-based PET reconstruction toolbox (occiput. io (32)), and its specific implementation for the Biograph mMR will be provided to interested users on request.

\section{CONCLUSION}

We presented and evaluated in vivo a comprehensive approach for self-gated MR motion modeling applied to respiratory motion compensation of PET and DCE-MRI data acquired simultaneously in an integrated PET/MR system. Fully registered, motion-corrected PET images and diagnostic DCE-MR images were obtained with negligible acquisition time prolongation compared with standard breath-hold techniques. Both the MR and the PET image quality and tracer uptake quantification were improved when compared with conventional methods. Furthermore, we demonstrated that respiratory motion correction of the PET data can also be performed on the basis of the MR data acquired in $1 \mathrm{~min}$, allowing for more flexible PET/MR scanning protocols.

\section{DISCLOSURE}

Funding for this work was provided by NIH grant 5R01-EB014894. We gratefully acknowledge the support of NVIDIA Corporation with the donation of the Titan X Pascal GPU used for this research. No other potential conflict of interest relevant to this article was reported.

\section{REFERENCES}

1. Catana C, Guimaraes AR, Rosen BR. PET and MR imaging: the odd couple or a match made in heaven? J Nucl Med. 2013;54:815-824.

2. Martinez-Möller A, Eiber M, Nekolla SG, et al. Workflow and scan protocol considerations for integrated whole-body PET/MRI in oncology. J Nucl Med. 2012;53:1415-1426.

3. Catalano OA, Masch WB, Catana C, et al. An overview of PET/MR, focused on clinical applications. Abdom Radiol (NY). 2017;42:631-644.

4. Li XA, Stepaniak C, Gore E. Technical and dosimetric aspects of respiratory gating using a pressure-sensor motion monitoring system. Med Phys. 2006;33:145-154.

5. Dawood M, Büther F, Lang N, et al. Respiratory gating in positron emission tomography: a quantitative comparison of different gating schemes. Med Phys. 2007;34:3067-3076.
6. Catana C. Motion Correction Options in PET/MRI. Semin Nucl Med. 2015;45: 212-223.

7. Thielemans K, Rathore S, Engbrant F, Razifar P. Device-less gating for PET/CT using PCA. Proc IEEE Nucl Sci Symp Med Imaging Conf. 2011;3904-3910.

8. Fürst S, Grimm R, Hong I, et al. Motion correction strategies for integrated PET/ MR. J Nucl Med. 2015;56:261-269.

9. McClelland JR, Hawkes DJ, Schaeffter T, et al. Respiratory motion models: a review. Med Image Anal. 2013;17:19-42.

10. King AP, Buerger C, Tsoumpas C, et al. Thoracic respiratory motion estimation from MRI using a statistical model and a 2-D image navigator. Med Image Anal. 2012;16:252-264.

11. Manber R, Thielemans K, Hutton BF, et al. Practical PET respiratory motion correction in clinical PET/MR. J Nucl Med. 2015;56:890-896.

12. Ozturk C, Derbyshire JA, McVeigh ER. Estimating motion from MRI data. Proc IEEE Inst Electr Electron Eng. 2003;9:1627-1648

13. Chun SY, Reese TG, Ouyang J, et al. MRI-based nonrigid motion correction in simultaneous PET/MRI. J Nucl Med. 2012;53:1284-1291.

14. Würslin C, Schmidt H, Martirosian P, et al. Respiratory motion correction in oncologic PET using T1-weighted MR imaging on a simultaneous whole-body PET/MR system. J Nucl Med. 2013;54:464-471.

15. Guérin B, Cho S, Chun SY, et al. Nonrigid PET motion compensation in the lower abdomen using simultaneous tagged-MRI and PET imaging. Med Phys. 2011;38:3025-3038.

16. Winkelmann S, Schaeffter T, Koehler T, et al. An optimal radial profile order based on the Golden Ratio for time-resolved MRI. IEEE Trans Med Imaging. 2007;26:68-76.

17. Buerger C, Clough RE, King AP, et al. Nonrigid motion modeling of the liver from 3-D undersampled self-gated golden-radial phase encoded MRI. IEEE Trans Med Imaging. 2012;31:805-815.

18. Grimm R, Fürst S, Souvatzoglou M, et al. Self-gated MRI motion modeling for respiratory motion compensation in integrated PET/MRI. Med Image Anal. 2015;19:110-120.

19. Chandarana H, Heacock L, Rakheja R, et al. Pulmonary nodules in patients with primary malignancy: comparison of hybrid PET/MR and PET/CT imaging. Radiology. 2013;268:874-881.

20. Chandarana H, Block TK, Rosenkrantz AB, et al. Free-breathing radial 3D fatsuppressed T1-weighted gradient echo sequence: a viable alternative for contrast-enhanced liver imaging in patients unable to suspend respiration. Invest Radiol. 2011;46:648-653.

21. Feng L, Grimm R, Block KT, et al. Golden-angle radial sparse parallel MRI: combination of compressed sensing, parallel imaging, and golden-angle radial sampling for fast and flexible dynamic volumetric MRI. Magn Reson Med. 2014;72:707-717.

22. Rank CM, Heußer T, Buzan MTA, et al. 4D Respiratory motion-compensated image reconstruction of free-breathing radial MR data with very high undersampling. Magn Reson Med. 2017;77:1170-1183.

23. Chandarana H, Feng L, Block KT, et al. Free-breathing contrast-enhanced multiphase MRI of the liver using a combination of compressed sensing, parallel imaging, and golden-angle radial sampling. Invest Radiol. 2013;48:10-16.

24. Pang J, Sharif B, Fan Z, et al. ECG and navigator-free 4D whole-heart coronary MRA for simultaneous visualization of cardiac anatomy and function. Magn Reson Med. 2014;72:1208-1217.

25. Feng L, Axel L, Chandarana H, et al. XD-GRASP: Golden-angle radial MRI with reconstruction of extra motion-state dimensions using compressed sensing. Magn Reson Med. 2016;75:775-788.

26. Fessler A, Sutton BP. Nonuniform fast Fourier transforms using min-max interpolation. IEEE Trans Signal Process. 2003;51:560-574.

27. Knoll F, Schwarzl A, Sodickson DK, et al. gpuNUFFT: an open-source GPU library for 3-D gridding with direct Matlab interface. Proc ISMRM. 2014;42:97-4308.

28. Klein S, Staring M, Pluim JPW, et al. elastix: a toolbox for intensity-based medical image registration. IEEE Trans Med Imaging. 2010;29:196-205.

29. Hudson HM, Larkin RS. Accelerated image reconstruction using ordered subsets of projection data. IEEE Trans Med Imaging. 1994;13:601-609.

30. Lamare F, Ledesma Carbayo MJ, Visvikis D, et al. List-mode-based reconstruction for respiratory motion correction in PET using non-rigid body transformations. Phys Med Biol. 2007;52:5187-5204.

31. Reader AJ, Verhaeghe J. 4D image reconstruction for emission tomography. Phys Med Biol. 2014;59:R371-R418.

32. Pedemonte S, Fuin N. Occiput.io. Tomography Lab website. Accessed June 28, 2018.

33. Kinahan PE, Karp JS. Figures of merit for comparing reconstruction algorithms with a volume-imaging PET scanner. Phys Med Biol. 1994;39:631-642. 